\title{
Bacillus subtilis RNase J1 endonuclease and 5' exonuclease activities in the turnover of $\triangle \mathrm{erm} C \mathrm{mRNA}$
}

\author{
SHIYI YAO, JOSH S. SHARP, ${ }^{1}$ and DAVID H. BECHHOFER \\ Department of Pharmacology and Systems Therapeutics, Mount Sinai School of Medicine of New York University, New York, New York 10029, \\ USA
}

\begin{abstract}
RNase J1, a ribonuclease with 5' exonuclease and endonuclease activities, is an important factor in Bacillus subtilis mRNA decay. A model for RNase $\mathrm{J1}$ endonuclease activity in mRNA turnover has RNase J1 binding to the $5^{\prime}$ end and tracking to a target site downstream, where it makes a decay-initiating cleavage. The upstream fragment from this cleavage is degraded by 3' exonucleases; the downstream fragment is degraded by RNase J1 5' exonuclease activity. Previously, $\triangle$ ermC mRNA was used to show $5^{\prime}$-end dependence of mRNA turnover. Here we used $\triangle$ erm $C$ mRNA to probe RNase J1-dependent degradation, and the results were consistent with aspects of the model. $\Delta$ erm $C$ mRNA showed increased stability in a mutant strain that contained a reduced level of RNase J1. In agreement with the tracking concept, insertion of a strong stem-loop structure at +65 resulted in increased stability. Weakening this stem-loop structure resulted in reversion to wild-type stability. RNA fragments containing the $3^{\prime}$ end were detected in a strain with reduced RNase J1 expression, but were undetectable in the wild type. The $5^{\prime}$ ends of these fragments mapped to the upstream side of predicted stem-loop structures, consistent with an impediment to RNase J1 $5^{\prime}$ exonuclease processivity. A $\Delta$ erm $C$ mRNA deletion analysis suggested that decay-initiating endonuclease cleavage could occur at several sites near the $3^{\prime}$ end. However, even in the absence of these sites, stability was further increased in a strain with reduced RNase J1, suggesting alternate pathways for decay that could include exonucleolytic decay from the $5^{\prime}$ end.
\end{abstract}

Keywords: Bacillus subtilis; mRNA decay; RNase J1; endonuclease cleavage; 5' exoribonuclease

\section{INTRODUCTION}

For some time, we have been using the mRNA encoded by the erythromycin-resistance gene, erm $C$, to study aspects of mRNA metabolism in Bacillus subtilis. ermC encodes a ribosomal RNA methylase that confers resistance to erythromycin (Em) by altering the Em binding site on the ribosome. Addition of low levels of Em results in induction of erm $C$ gene expression, and this occurs at the translational level (Dubnau 1984; Weisblum 1985). In the translational attenuation mechanism, addition of Em results in ribosome stalling, now known to occur after incorporation of amino acid 9 of the ermC leader peptide coding sequence (CDS) (Vazquez-Laslop et al. 2008; Yao et al. 2008). This, in turn,

\footnotetext{
${ }^{1}$ Present address: Children's Hospital Boston, Department of Infectious Diseases, 300 Longwood Avenue, Enders Building, Room 750.7, Boston, MA 02115, USA.

Reprint requests to: David H. Bechhofer, Department of Pharmacology and Systems Therapeutics, Mount Sinai School of Medicine of New York University, New York, NY 10029, USA; e-mail: david.bechhofer@mssm.edu; fax: (212) 996-7214.

Article published online ahead of print. Article and publication date are at http://www.rnajournal.org/cgi/doi/10.1261/rna.1749109.
}

causes the opening of an inhibitory RNA secondary structure, allowing high-level methylase translation. Our initial interest in the ermC message came from the observation that induction of ermC mRNA translation was accompanied by strong stabilization of erm $C$ mRNA. The stabilization of erm $C$ mRNA in the presence of Em was attributed to the protective effect of an Em-bound ribosome that is stalled in the $5^{\prime}$-proximal leader peptide coding sequence (Bechhofer and Dubnau 1987; Bechhofer and Zen 1989; DiMari and Bechhofer 1993). More recently, we have worked with a deleted version of erm $C$, called $\Delta e r m C$ (Fig. 1A), which has the first 16 codons of the leader peptide CDS fused in-frame to the carboxy-terminal portion of the ermC methylase CDS, giving a $\sim 260$ nucleotide (nt) mRNA that includes a 62-amino acid CDS (Drider et al. 2002). The small size, high level of transcription, and induced stability of $\Delta e r m C$ mRNA make it a useful model for studying mRNA decay (Sharp and Bechhofer 2003, 2005).

We have observed that, upon Em-induced ribosome stalling, $\Delta$ erm $C$ mRNA undergoes a processing event that results in the accumulation of a 215-nt RNA fragment whose $5^{\prime}$ end maps to +45 (Fig. $1 \mathrm{~A}$ ), which is the beginning 


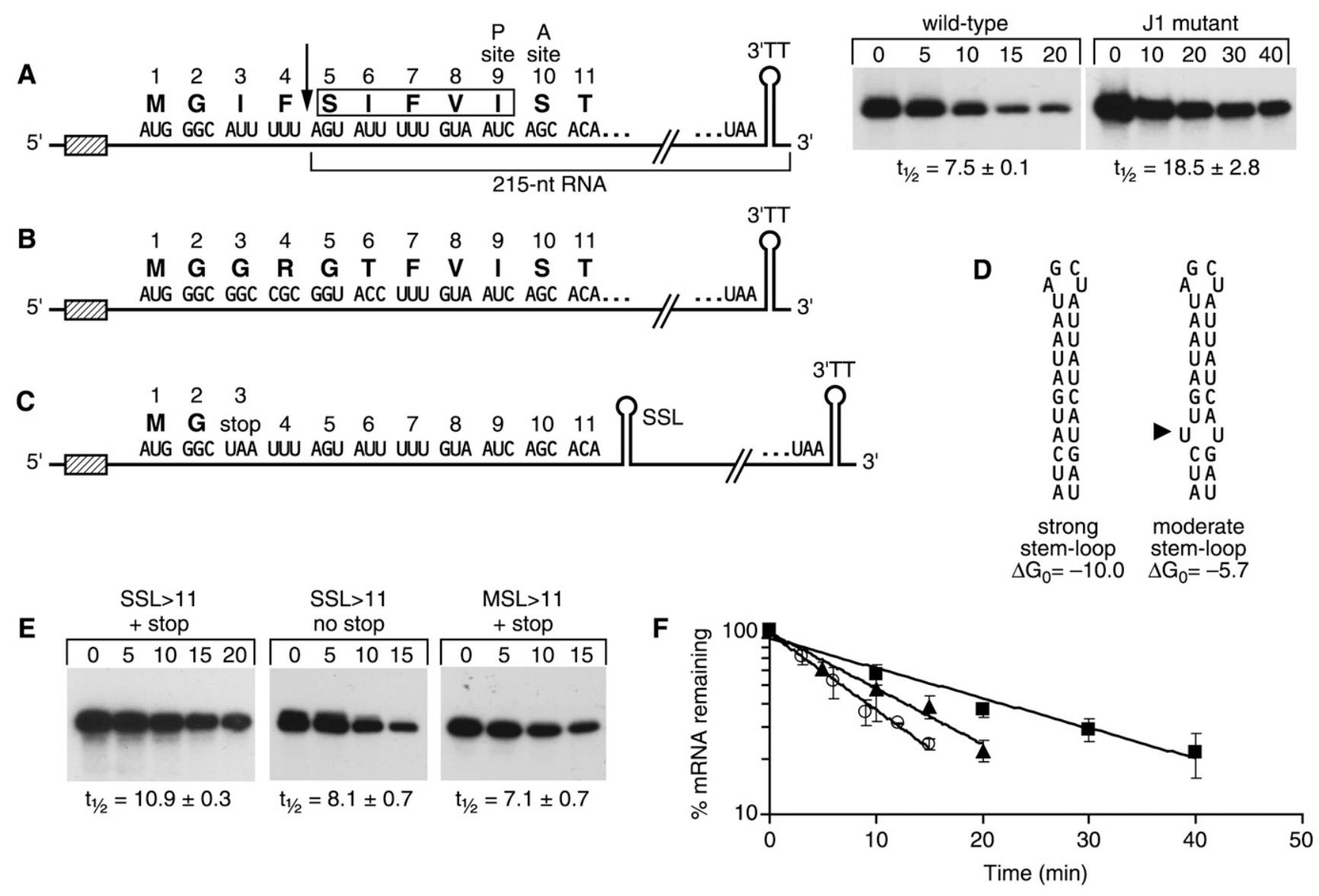

FIGURE 1. Diagrams of $\Delta$ erm $C$ mRNA wild type and mutants. (A) Wild-type $\Delta$ erm $C$ mRNA showing ribosome binding site (hatched box near $5^{\prime}$ end), site of RNase J1 cleavage upon Em-induced ribosome stalling (downward arrow), amino acids required for ribosome stalling (boxed), codons in the ribosome P site and A site when ribosome stalling occurs, transcription terminator structure (3'TT), and extent of 215-nt RNA observed upon ribosome stalling. (Note that this latter processing product was formerly called the "209-nt RNA" [Drider et al. 2002; Yao et al. 2008], since the size of full-length erm $C$ mRNA was taken as $254 \mathrm{nt}$. We now estimate the size of full-length $\Delta$ erm $C$ mRNA to be $260 \mathrm{nt}$; hence the processed product is a 215-nt RNA.) At right are Northern blot analyses of $\Delta$ ermC mRNA decay in wild-type and RNase J1 conditional mutant strains, grown in the presence of IPTG. Time indicated above each lane is minutes after rifampicin addition. The half-life value \pm standard deviation, which was the average of at least three determinations, is shown below the blots. As a control for the amount of RNA loaded, blots were stripped and reprobed with a $5 \mathrm{~S}$ rRNA probe. (B) Mutation of AU-rich $\Delta$ ermC codons to GC-rich codons. $(C) \Delta e r m C$ mRNA with stop after codon 2 and strong stem-loop inserted after codon 11. (D) Nucleotide sequence and predicted structure of strong and moderate stem-loops inserted after codon 11. Arrowhead points to single nucleotide change that substantially weakens the structure. Free energies $\left(\mathrm{kcal} \mathrm{mol}^{-1}\right)$ of the predicted structures are at the bottom. (E) Northern blot analysis of decay of constructs with strong or moderate stem-loop insert and in the presence and absence of translation. $(F)$ Plot of half-life data (average of three experiments) for $\Delta$ erm $C$ in wild-type strain (open circles), $\Delta$ erm $C$ in RNase J1 mutant strain (closed squares), and $\Delta e r m C$ SSL $>11$ (closed triangles).

of codon 5 of the leader peptide CDS (Drider et al. 2002). We showed recently that this processing event, whose product is only detectable when Em is added and ribosome stalling occurs, was dependent on RNase $\mathrm{J} 1$ (Yao et al. 2008). Several experiments provided strong evidence that processing of $\Delta$ erm $C$ mRNA at the +45 site was the result of RNase J1 endonucleolytic cleavage, rather than exonuclease decay from the $5^{\prime}$ end up to the stalled ribosome. It is not known if cleavage at this site only occurs when a ribosome stalls or if cleavage occurs constitutively, but the downstream cleavage product is too unstable to be detected unless a stalled ribosome is present to protect its $5^{\prime}$ end.

RNase J1 was recently identified as a B. subtilis ribonuclease that has both endonuclease and $5^{\prime}$-to- $3^{\prime}$ exonuclease activities (Even et al. 2005; Britton et al. 2007; Mathy et al. 2007; Deikus et al. 2008; Li de la Sierra-Gallay et al. 2008). RNase $\mathrm{J} 1$ is an essential enzyme and is believed to be a major player in mRNA decay in B. subtilis. A similar ribonuclease, RNase J2, is not essential. A strain in which RNase J1 is under control of an IPTG-inducible promoter contains a reduced level of RNase J1 when grown in the presence of IPTG. This strain shows a small but significant increase in overall mRNA half-life (Even et al. 2005). A recent microarray study showed that the level of many RNAs is affected in a strain that is deleted for RNase J2 and that has reduced expression of RNase J1 (Mader et al. 2008). RNase J1 5'-to-3' exonuclease activity requires a monophosphate or hydroxyl $5^{\prime}$ end and is inhibited by 
a triphosphate 5' end (Mathy et al. 2007; Deikus et al. 2008; Li de la Sierra-Gallay et al. 2008). On the other hand, the endonuclease activity of RNase $\mathrm{J} 1$ is insensitive to the phosphorylation state of the $5^{\prime}$ end (Deikus et al. 2008; Li de la Sierra-Gallay et al. 2008).

Current models of mRNA decay in B. subtilis (Bechhofer 2009) propose that RNase J1 can initiate mRNA decay in one of two ways: RNase J1 may bind to the $5^{\prime}$ end and degrade mRNA processively in the $5^{\prime}$-to- $3^{\prime}$ direction. Presumably this activity is preceded by conversion of the $5^{\prime}$ triphosphate nucleoside of the transcription product into a $5^{\prime}$ monophosphate nucleoside, as has been proposed for the initiation of RNase E activity in E. coli (Celesnik et al. 2007; Deana et al. 2008). Alternatively, RNase J1 binds to the $5^{\prime}$ end and tracks to an endonucleolytic target site (Condon 2007). Cleavage at this site results in an upstream fragment that has an unprotected $3^{\prime}$ end and that is degraded processively by $3^{\prime}$-to- $5^{\prime}$ exonucleases, and a downstream fragment that is the target of further RNase J1 endonuclease cleavages and/or RNase J1 5'-to-3' exonuclease activity. In this study, we examined involvement of RNase J1 in $\Delta e r m C$ mRNA decay.

\section{RESULTS}

\section{AermC mRNA decay is RNase J1 dependent}

To determine the dependence of $\Delta$ erm $C$ mRNA decay on RNase J1, a strain was constructed that carried a $\Delta e r m C$ gene on a high-copy plasmid and that conditionally expressed RNase J1. In this strain, RNase J1 transcription is under the control of an IPTG-inducible promoter, and it has been shown that addition of IPTG results in a level of RNase J1 that is approximately fivefold lower than in the wild-type strain (Daou-Chabo et al. 2009). $\Delta e r m C$ mRNA half-life measurements were performed in the presence of IPTG to avoid conditions of severe growth retardation that occur in the absence of IPTG, where the essential RNase J1 is present at severely reduced levels and where one might expect an effect on mRNA half-life due to slow growth rate. Northern blot analysis of $\Delta e r m C$ mRNA decay in a wildtype versus RNase J1 conditional strain showed a 2.5 -fold increase in half-life under conditions of reduced RNase J1 (Fig. 1A, right, Fig. 1F). This result suggested that decay of $\Delta$ erm $C$ mRNA is largely dependent on RNase J1 activity, as even a reduction (and not elimination) of this function substantially increased mRNA half-life.

We next determined whether cleavage at the +45 site, which we detect only in the presence of Em-induced ribosome stalling (Drider et al. 2002; Yao et al. 2008), was a factor in $\Delta e r m C$ mRNA half-life. A $\Delta e r m C$ mRNA derivative was made in which the AU-rich nucleotide sequence at the RNase $\mathrm{J} 1$ cleavage site was mutated to a GC-rich sequence (Fig. 1B). The RNase J1 endonuclease cleavage sites known to date all occur in AU-rich sequences
(Bechhofer 2009). We reasoned that, if RNase J1 cleavage at this site was constitutive and was involved in initiating decay, then altering the site such that RNase J1 would no longer recognize it might result in a longer $\Delta$ erm $C$ mRNA half-life. A similar sequence change at an RNase J1 target site in $\operatorname{trp}$ leader RNA resulted in a fourfold increase in RNA stability (Deikus and Bechhofer 2007). The effect of the GC-rich sequence on RNase J1 recognition was confirmed by in vitro analysis of RNase J1 cleavage of $5^{\prime}$-endlabeled $\Delta e r m C$ mRNA (data not shown). A band representing cleavage in the AU-rich region around nucleotide 45 was observed for wild-type $\Delta e r m C$ mRNA, but was absent for GC-rich mutant mRNA. Northern blot analysis (data not shown) of the decay of RNA encoded by the construct with the GC-rich sequence in codons 3-6 showed a half-life of $6.8 \pm 0.4 \mathrm{~min}$, which was barely significantly different from the wild-type half life of $7.5 \pm 0.1 \mathrm{~min}(P$-value $=$ 0.044). Thus, RNase J1 cleavage at the +45 site is not a factor in $\Delta e r m C$ mRNA decay, and there are likely target sites further downstream on the $\Delta e r m C$ sequence.

\section{Evidence for RNase J1 tracking from the $5^{\prime}$ end}

Since we had demonstrated earlier that $\Delta e r m C$ mRNA is stabilized by the presence of secondary structure at the $5^{\prime}$ end (Sharp and Bechhofer 2005), it was reasonable to assume that RNase J1 binds at the $5^{\prime}$ end and then tracks to a target site downstream, as has been suggested for initiation of $B$. subtilis mRNA decay generally (Condon 2003). We tested whether the presence of a strong secondary structure in the $\Delta e r m C$ coding sequence affected mRNA half-life, as this could slow down RNase J1 tracking. A construct was made that had a strong stem-loop inserted after codon 11 (Fig. 1C). This construct was named SSL $>11$ (strong stem-loop after codon 11). The stem-loop sequence was the $5^{\prime}$-terminal stem-loop structure of a mutant $m d r$ (or $b m r 3$ ) RNA (Fig. 1D), which was shown to increase $m d r$ mRNA stability at least fourfold when located at the $5^{\prime}$ end (Ohki and Tateno 2004). The SSL $>11$ construct was in the context of a stop codon after codon 2, to insure that the strong stem-loop was not unwound by ribosome transit. In a Northern blot analysis, SSL $>11$ RNA was found to be significantly more stable than wild-type $\Delta e r m C$ mRNA, with a half-life of $10.9 \mathrm{~min}$ (Fig. 1E,F). Since we had found earlier that the stability of $\Delta$ erm $C$ mRNA was unaffected by the presence of a stop after codon 2 (Sharp and Bechhofer 2003), the increased stability of SSL $>11$ RNA was attributed to the stem-loop structure insert, despite it being located relatively far from the $5^{\prime}$ end. This result suggested that the presence of the strong stem-loop structure hindered RNase J1 tracking, causing a delay of downstream target site binding and decay-initiating cleavage. Two predictions of this model were tested.

First, the absence of a stop after codon 2 might result in a wild-type half-life, as the translating ribosome could melt 
the strong structure. An SSL $>11$ construct was made that did not contain the stop after codon 2. As shown in Figure $1 \mathrm{E}$, the half-life of this construct was $8.1 \mathrm{~min}$, similar to that of wild-type $\Delta e r m C$ mRNA $(P$-value $=0.12)$.

Second, replacement of the strong stem-loop structure after codon 11 with a moderately strong structure should decrease half-life, as there would be less inhibition of tracking from the $5^{\prime}$ end. A construct was made that was almost identical to SSL $>11$, but that had a single nucleotide change in the stem sequence that lowered the stability of the predicted structure substantially (Fig. 1D). This construct was designated MSL $>11$ (moderate stem-loop after codon 11). The RNA encoded by this construct gave a half-life that was $7.1 \mathrm{~min}$, similar to that of wild-type $\Delta e r m C$ mRNA (Fig. 1E). Furthermore, the stability of MSL $>11$ RNA was only marginally sensitive to the stop codon after 2 . When the stop codon was changed to the wild-type isoleucine codon, the half-life was 6.3 min (data not shown).

\section{Detection of 3 '-end-containing decay intermediates in the RNase J1 mutant strain}

Our working hypothesis was that initiation of $\Delta \mathrm{ermC}$ mRNA decay is dependent on cleavage at an RNase J1 target downstream from the site SSL $>11$ insertion, which generates unstable upstream and downstream RNA fragments. The upstream product of such cleavage would be expected to be rapidly degraded by $3^{\prime}$-to- $5^{\prime}$ exonuclease activity. The downstream product of such cleavage would be expected to be rapidly degraded by RNase J1.

We attempted to detect downstream products in the RNase J1 conditional mutant strain. RNA isolated from the wild-type strain and from an RNase J1 conditional mutant strain grown in the absence of IPTG (i.e., severely reduced RNase J1 levels) was probed with a labeled oligonucleotide that was complementary to nucleotides 205-240 near the 3' end of $\Delta e r m C$ mRNA. The results in Figure 2A $(\Delta e r m C$ blot) show that this probe did not detect any decay intermediates in the wild-type strain. In the RNase J1 mutant strain, however, two prominent sets of bands, designated " $\sim 140$ " and " $~ 85$," in addition to several less prominent bands, were detected (see the schematic of the prominent bands in Fig. 2C). We postulated that these bands were the result of endonuclease cleavage followed by limited $5^{\prime}$ exonuclease processing. In the wild-type strain, which had a much higher level of RNase J1, these fragments were rapidly degraded by the $5^{\prime}$-to- $3^{\prime}$ exonuclease activity of RNase J1, as we have shown previously that $3^{\prime}$-terminal, structured fragments can be degraded by RNase J1 (Deikus et al. 2008). In the RNase J1 mutant strain, with a sharply reduced level of RNase J1, these fragments were detectable since they were not degraded as rapidly. We assumed that the $3^{\prime}$ end of these fragments was protected from $3^{\prime}$-to- $5^{\prime}$ exonuclease decay by the extremely stable $\Delta e r m C$ transcription terminator stem-loop, which has a $\Delta G_{0}$ of $-16.2 \mathrm{kcal}$ $\mathrm{mol}^{-1}$. In data not shown, the prominent bands at $\sim 140$ and $\sim 85$ nt were also detectable in the RNase J1 conditional strain grown in the presence of IPTG (i.e., only a fivefold reduction in the RNase J1 level), but not nearly as strongly as in the same strain grown in the absence of IPTG.

Additional Northern blotting was performed using a high-resolution gel to resolve the multiple RNA fragments that constituted the two major bands detected on the low-resolution gel in Figure 2A. This analysis (Fig. 2B, lane $\Delta \mathrm{e}$ ) showed that the two sets of bands detected by the $\Delta e r m C 3^{\prime}$-terminal probe ran between nucleotides 128 and 143 and between nucleotides 80 and 95. These corresponded to RNAs with 5' ends in the regions of nucleotides 117-132 and 165-180 (Fig. 2C).

To understand why these fragments were accumulating in the strain with a reduced level of RNase J1, we looked for secondary structure near the $5^{\prime}$ ends of these fragments. The $\Delta e r m C$ sequence was analyzed by the Zuker mfold version 3.3 program, using 50 -nt increments $10 \mathrm{nt}$ apart, i.e., 91$140,101-150,111-160$, etc. We used an arbitrary cutoff of $\Delta G_{0}<-3.0 \mathrm{kcal} \mathrm{mol}^{-1}$ for consideration as a stable structure. Only three predicted structures with significantly low free energy were predicted. The first two of these, from nucleotides 125-150 and 143-184, contained overlapping sequences, so we chose the sequence from 125-150, which was considerably more stable with a $\Delta G_{0}=-6.4 \mathrm{kcal}$ $\mathrm{mol}^{-1}$. The other sequence was from 167-202, with a $\Delta G_{0}=$ $-5.1 \mathrm{kcal} \mathrm{mol}^{-1}$. The sequence of these predicted secondary structures, which were designated eSL1 (ermC stem-loop 1) and eSL2, are shown in Figure 2C. We surmised that endonuclease cleavage at sites upstream of these structures (Fig. 2C, downward open arrows) would, under conditions of low RNase J1 activity, result in the accumulation of decay intermediates with $5^{\prime}$ ends that map to the upstream side of these structures. To test this, the $3^{\prime}$ stem sequence of eSL2 was changed to a NotI sequence (Fig. 2C), which eliminated eSL2 secondary structure. Northern blot analysis of this RNA on a low-resolution gel (Fig. 2A, NotI blot) showed that this change had no effect on the $\sim 140$-nt band, but resulted in loss of the $\sim 85$-nt band and appearance of a 70-nt band. Results from a high-resolution gel (Fig. 2B, NotI lane) showed that the upstream fragments with $5^{\prime}$ ends in the region from 117-132 were unchanged by this mutation whereas the downstream fragments with $5^{\prime}$ ends from 165-180 were no longer present. Instead, fragments that had 5' ends mapping from 187-200 were observed (see Discussion). In a separate construct, we made a change in the $5^{\prime}$ stem sequence of eSL1 to give a ClaI site (Fig. 2C), which eliminated eSL1 secondary structure. The low-resolution gel in Figure 2A (ClaI blot) showed loss of the $\sim 140$-nt band and retention of the $\sim 85$-nt band. These results were consistent with RNase J1 endonucleolytic cleavage upstream of eSL1 and eSL2, followed by RNase J1 5' -to-3' exonucleolytic degradation being hindered by the eSL1 and eSL2 secondary structures. 


\section{Pattern of 3'-proximal decay intermediates in the absence of ribosome transit}

We tested whether detection of the decay intermediates in the strain with reduced RNase J1 levels was dependent on ribosome transit. Decay intermediates from a $\Delta$ erm $C$ construct with a stop after codon 2 were analyzed using the $3^{\prime}$-proximal probe. As shown in Figure 3A, the steadystate pattern of decay intermediates in the RNase J1 mutant background was identical for translated and untranslated $\Delta e r m C$ mRNA. Thus, the putative endonuclease cleavages in the body of the message were not dependent on ribosome flow.

\section{Differential accumulation of cleavage products}

According to our hypothesis, the detection of the $3^{\prime}$-end-containing fragments in the RNase J1 conditional mutant strain was possible because of slower decay of these fragments due to a reduction in the level of RNase J1 5'-to-3' exonuclease activity. However, long exposure times were required to observe these decay intermediates, suggesting that they do not accumulate. These decay intermediates differed from the 215nt RNA seen upon RNase J1 cleavage at +45 (Fig. 1A), which accumulates with time after rifampicin addition and is easy to detect (Drider et al. 2002; Yao et al. 2008). We analyzed the decay of processing intermediates in the RNase $\mathrm{J} 1$ conditional mutant strain (grown in the presence of IPTG), after addition of Em and subsequent addition of rifampicin. As can be seen in the shorter exposure in Figure 3B, the amount of the 215-nt fragment increased with time, as the full-length RNA was processed by RNase J1 endonuclease cleavage at +45 . (The 215-nt RNA accumulates to higher levels in a wild-type strain that has a higher level of RNase J1.) By contrast, the amount of the two sets of bands that represent 3 '-end-containing fragments generated by downstream processing decreased with time (Fig. 3B, long exposure). Most likely, the 215-nt fragment is protected from further $5^{\prime}$-to-3' degradation due to the stalled ribosome at its $5^{\prime}$ end, whereas the downstream fragments are subject to ongoing $5^{\prime}$-to- $3^{\prime}$ decay.

\section{Deletion analysis}

If endonuclease cleavage at RNase J1 target sites in the body of $\triangle$ ermC mRNA was responsible for its decay, then

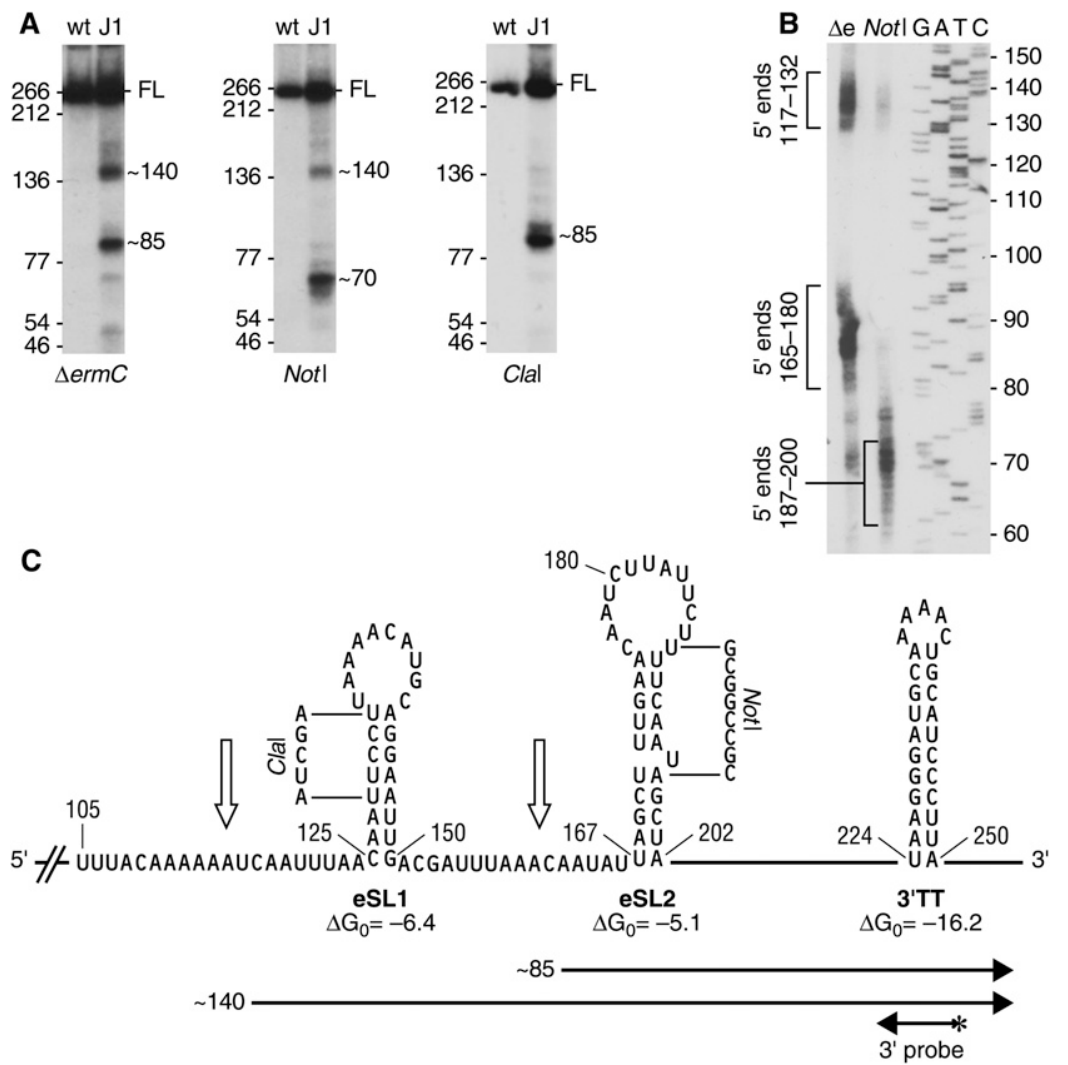

FIGURE 2. Northern blot analysis of 3 '-end-containing decay intermediates. (A) Steady-state pattern of $3^{\prime}$-end-containing decay intermediates in the wild-type (wt) and RNase J1 conditional mutant strain (J1), grown in the absence of IPTG. Blots of wild-type $\Delta$ ermC RNA, NotI mutant RNA, and ClaI mutant RNA are shown, as indicated. On the left are the sizes (nucleotides) of $5^{\prime}$-end-labeled pSE420 TaqI DNA fragments (Brosius 1992) run in a parallel lane. On the right, the migration of full-length (FL) RNA and major decay intermediate bands are indicated. $(B)$ High-resolution Northern blot analysis of $\Delta e r m C$ wild-type $(\Delta \mathrm{e})$ and NotI construct RNA. ladder was generated from M13mp18 single-stranded DNA. Sizes of the sequencing ladder bands are indicated on the right. $(C)$ Partial nucleotide sequence of the downstream portion of $\Delta \mathrm{ermC}$ mRNA, showing $\Delta$ erm $C$ stem-loops 1 and 2 and their predicted free energies, as well as location of NotI and ClaI mutations. Approximate $5^{\prime}$ ends of the two groups of decay intermediates are shown schematically below, as well as the location of the complementary $3^{\prime}$ probe.

deletion of these sites should result in a more stable $\Delta$ erm $C$ mRNA. Thus, we analyzed the stability of RNAs encoded by a number of $\triangle e r m C$ deletion mutants. The half-life of wildtype $\Delta$ erm $C$ mRNA in these experiments was about $7 \mathrm{~min}$ (Fig. 4A). Deletion of small regions starting from near the $3^{\prime}$ end (Fig. 4B-D) had no significant effect on $\Delta$ ermC mRNA half-life. Even deletion of a 95-nt segment that included both regions in which endonuclease cleavages were thought to occur (Fig. 4E) did not significantly affect $\Delta e r m C$ mRNA half-life. These results suggested that elimination of one or more endonuclease target sites was insufficient to confer increased stability, and perhaps additional target sites were still present. Interestingly, deletion of a 65-nt segment that was closer to the $3^{\prime}$ end (Fig. $4 \mathrm{~F})$ resulted in a significant, $40 \%$ increase in half-life, despite the fact that the upstream target site should still be present in this construct. 

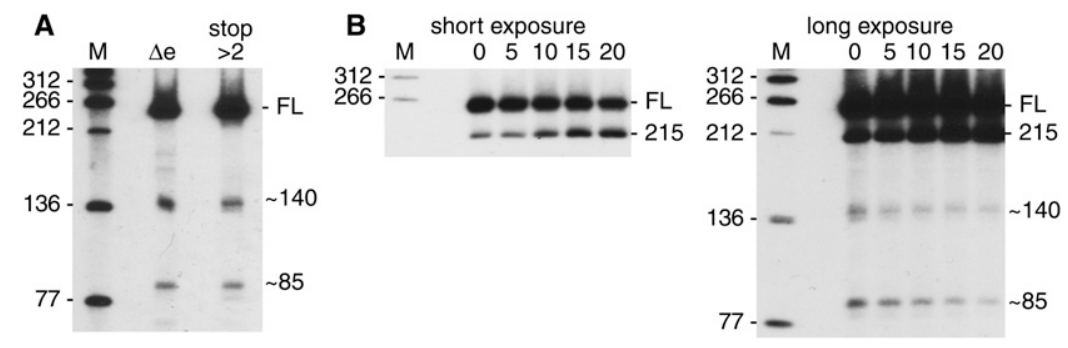

FIGURE 3. Northern blot analysis of $(A) 3^{\prime}$-end-containing decay intermediates detected with $(\Delta e)$ and without (stop $>2$ ) translation, and $(B)$ processed RNA and decay intermediates in the presence of Em-induced ribosome stalling.

When a large, 129-nt deletion was made that included nucleotides 87-215 (Fig. 4G), the half-life of $\Delta e r m C$ mRNA jumped twofold to about $15 \mathrm{~min}$. The increase in stability of this RNA could have been due to its small size, which was only $131 \mathrm{nt}$. Two controls for RNA size were constructed, in which deletions of the same size were made elsewhere in the RNA (Fig. $4 \mathrm{H}, \mathrm{I})$. In both cases, the $\Delta$ erm $C$ mRNA half-life was similar to that of wild type. Taken together with the results obtained with 3 '-end-containing decay intermediates (Fig. 2), the deletion analysis indicated that there were at least three target sites for initiation of decay by endonuclease cleavage (Figure 4A, arrows), and that elimination of these three targets had a profound effect on mRNA half-life. A prediction of this conclusion was that insertion of an endonuclease target site into the stable construct shown in Figure $4 \mathrm{G}$ should result in destabilization. This was the case: insertion of nucleotides 151-215 into an upstream region of the stable deletion mutant (Fig. 4J) resulted in a half-life that was actually somewhat shorter than wild type.
Finally, the stability of the 129-nt deletion construct (Fig. 4G) was tested in the RNase J1 conditional mutant. If, in fact, the deletion eliminated all RNase J1 recognition sites, and decay was proceeding by an RNase J1-independent pathway, we expected that, unlike the case with wild-type $\Delta e r m C$ mRNA (Fig. $1 \mathrm{~A})$, the reduction in RNase J1 levels should not affect stability. Surprisingly, we found that the stability of the RNA with the large deletion was even greater in the RNase J1 mutant (Fig. 4G, right), with a half-life of more than $40 \mathrm{~min}$. This suggested that RNase J1 is involved even in decay of the RNA encoded by the 129-nt deletion construct, perhaps by a $5^{\prime}$-to- $3^{\prime}$ exonucleolytic pathway or by cleavage at sites in the upstream half of the RNA.

\section{DISCUSSION}

Although the $\Delta e r m C$ gene is an artificial construct, it has all the elements of a small mRNA, and, we believe, can be used as a model to study mRNA decay. It should be noted that the $\Delta e r m C$ mRNA sequence has a low GC content (27\%), even lower than that of $B$. subtilis (43\%), which is characterized as a "low GC" organism. It is therefore possible that $\Delta e r m C$ mRNA contains a higher than average density of target sites for RNase J1, which appears to cleave preferentially at AU-rich sequences (Bechhofer 2009).

Decay intermediates are normally difficult to detect. Once the initial, rate-determining step in mRNA decay

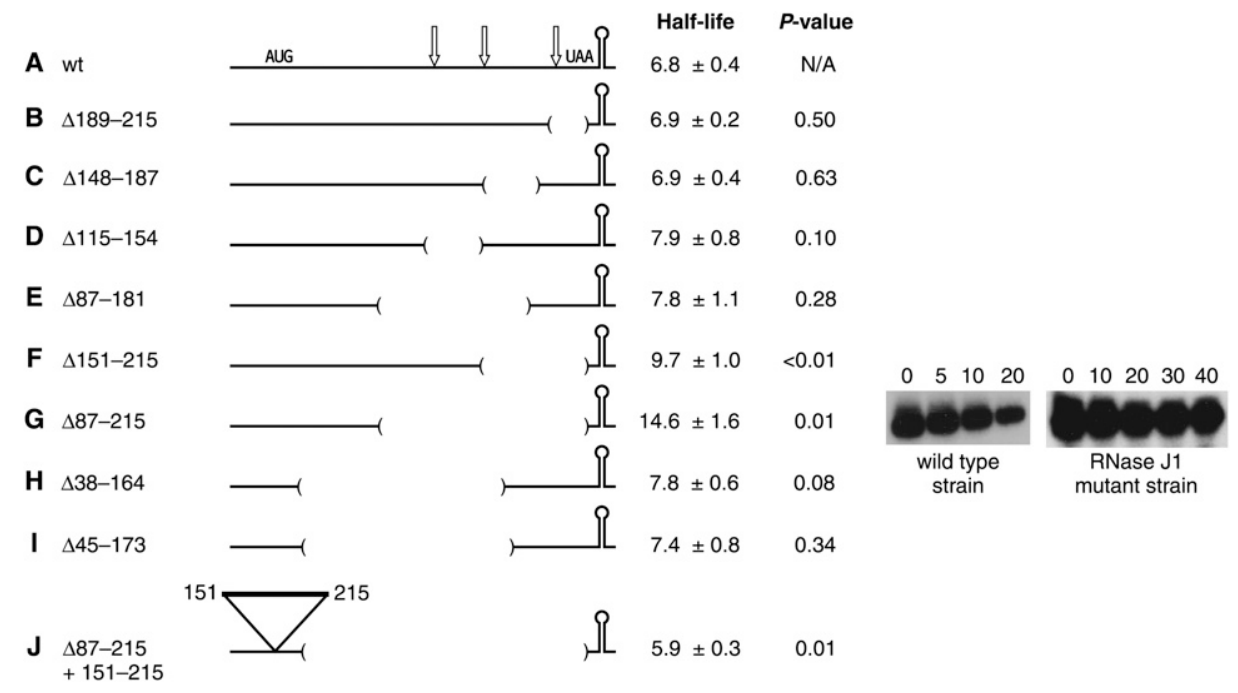

FIGURE 4. Deletion analysis of $\Delta e r m C$ mRNA. Schematic diagram of $(A)$ wild-type (wt) $\Delta e r m C$ mRNA is shown at the top, with downward arrows indicating putative sites of RNase J1 endonucleolytic cleavage. Half-lives (average of at least three determinations) are shown at right, together with $P$-value relative to wild-type half-life. For the deletion constructs $B-J$, the extent of the deletion in nucleotides is indicated at the left and represented by parentheses on the schematic diagrams. Construct $J$ contained an insertion of nucleotides 151-215 into the 5' -proximal region of construct $G$. Northern blot analysis of construct $G$ half-life, in wild-type and RNase J1 conditional mutant strains (grown in the presence of IPTG), is shown at the right of the construct $G$ schematic. 
occurs, RNA fragments that are generated in the course of complete turnover are rapidly degraded. The use of $\Delta \mathrm{ermC}$ mRNA enabled the analysis of intermediates in the process of mRNA decay. With relatively long exposure times, we were able to detect $3^{\prime}$-end-containing $\Delta e r m C$ mRNA decay intermediates in the RNase J1 conditional mutant strain. The combination of $\Delta e r m C$ on a high-copy plasmid, transcription from a strong promoter, secondary structure to slow rapid $5^{\prime}$-to- $3^{\prime}$ decay, and a strain with reduced RNase J1 levels made this detection possible.

We found that $\Delta e r m C$ mRNA half-life doubled even under conditions where RNase J1 was still present, but at reduced levels (Fig. 1A), which indicated strongly that $\Delta e r m C$ mRNA decay is dependent on RNase J1 activity. An important goal of this study was to determine which of the two ribonuclease activities of RNase $\mathrm{J} 1,5^{\prime}$ exonuclease or endonuclease (or both) is (are) involved in decay of a particular mRNA. Several findings argue against $\Delta$ erm $C$ mRNA being degraded solely exonucleolytically from the $5^{\prime}$ end (which would initiate after $5^{\prime}$ pyrophosphate removal). First, we found that the presence of a strong stem-loop structure at nucleotide +65 , which presumably is too far downstream to affect the $5^{\prime}$ end as a substrate for RNase $\mathrm{J} 15^{\prime}$ exonuclease activity, resulted in increased stability (Fig. 1D). Even if $5^{\prime}$-to-3' exonuclease processivity would be slowed by the inserted stem-loop, this would not affect a half-life measurement that is dependent on the amount of full-length RNA remaining. Second, if $\Delta e r m C$ mRNA were degraded only by an exonucleolytic pathway, there should be no effect of deletions situated well downstream from the $5^{\prime}$ end. We found, however, that a 129-nt deletion starting at nucleotide 87 caused a substantial increase in halflife (Fig. 4G). Furthermore, insertion of a 65-nt segment from the downstream half of $\triangle e r m C$ mRNA, in which we mapped possible endonuclease cleavage sites, resulted in destabilization of the 129-nt deletion construct (Fig. 4J), which is also inconsistent with attack solely from the $5^{\prime}$ end. We conclude, therefore, that $\Delta e r m C$ mRNA decay is, at least to some extent, dependent on endonucleolytic cleavage. The stability conferred by $5^{\prime}$-terminal stem-loop structure (Sharp and Bechhofer 2005) is presumably due to interference with RNase J1 binding at the $5^{\prime}$ end, which is required not only for exonuclease activity, but also for initiation of tracking to downstream target sites.

Although we have not formally proved that the endonuclease activity involved in $\triangle e r m C$ mRNA decay is that of RNase J1, this is a reasonable interpretation of our results. The demonstrated $5^{\prime}$-end-dependence of $\Delta e r m C$ mRNA stability (Sharp and Bechhofer 2005) suggests involvement of RNase J1, whose 5' exonuclease activity is known to be $5^{\prime}$-end dependent and perhaps its endonuclease activity requires a $5^{\prime}$ end as well. An earlier review of mRNA decay in B. subtilis (Condon 2003) compared the $5^{\prime}$-enddependence of decay in this organism versus $5^{\prime}$-enddependence of RNase E in E. coli. From many studies, a model was suggested in which E. coli RNase E binds to the $5^{\prime}$ end of a message, but then loops around potential roadblocks (ribosomes, bound protein, RNA secondary structure) to its endonuclease target sites, whereas the similarly functioning $5^{\prime}$-dependent endonuclease in B. subtilis (not known to be RNase $\mathrm{J} 1$ at the time) depends on tracking on the mRNA in the $5^{\prime}$-to- $3^{\prime}$ direction to reach its target cleavage site. The observation of mRNA stabilization by an internal strong stem-loop structure (the SSL $>11$ RNA) (Fig. 1C) is consistent with this model. When this structure was disrupted, either by ribosome transit or by introduction of a destabilizing base change, the half-life reverted to wild type (Fig. 1E). Thus, we hypothesize that RNase J1 binds to the $5^{\prime}$ end of $\Delta e r m C$ (or to a site close to the $5^{\prime}$ end) and then tracks downstream until it reaches a target site. Insertion of the SSL after codon 11 hinders RNase J1 tracking to downstream target sites, which is necessary for initiation of decay. From the fact that the stabilizing effect of SSL $>11$ was abolished by translation of the message (Fig. 1E), we surmise that translated mRNAs have naturally few roadblocks to RNase J1 tracking to downstream endonuclease target sites. More likely, the regulation of mRNA stability by interference with RNase J1 activity occurs at the binding step at the $5^{\prime}$ end, upstream of ribosome flow. We have found that, when the same stem-loop structure that is present in the SSL $>11$ construct was placed at the $5^{\prime}$ end of an RNA that has a $5^{\prime}$-proximal RNase J1 target site, cleavage by RNase $\mathrm{J} 1$ at this site was virtually abolished (Yao and Bechhofer 2009).

Our earlier finding of RNase J1 cleavage at the +45 site (Drider et al. 2002; Yao et al. 2008) was shown here not to be a factor in constitutive mRNA decay (based on results with the construct shown in Fig. 1B). Rather, RNase J1 cleavage at +45 is likely a consequence of Em-induced ribosome stalling. RNases J1 and J2 were originally isolated from a high salt ribosomal wash fraction (Even et al. 2005), and biochemical studies have shown that RNase J1 colocalizes with ribosomes (Hunt et al. 2006), suggesting a strong interaction. Thus, the observed association of RNase J1 with ribosomes fits well with the notion of RNase J1-mediated cleavage at the +45 site in response to ribosome stalling. However, it is clear that RNase J1 endonuclease cleavage is not completely ribosome dependent, since such cleavage occurs in trp leader RNA (Deikus and Bechhofer 2007; Deikus et al. 2008) and in small cytoplasmic RNA (Yao et al. 2007), which are untranslated RNAs. Here, too, we showed that the pattern of $\Delta$ ermC mRNA decay intermediates, which we suggest arises following RNase J1 endonuclease cleavage, was the same in the presence and absence of ribosome flow (Fig. 3A). Thus, ribosome-dependent cleavage by RNase $\mathrm{J} 1$ at the +45 site is likely a special case of endonuclease cleavage in response to an urgent situation (ribosome stalling), but endonuclease activity of RNase J1 is generally not ribosome dependent.

We reported earlier that fragments containing the 3'-terminal sequence are detectable in an RNase J1 
conditional mutant (Deikus et al. 2008). These were fragments that contained the transcription terminator sequence, and, as such, we could not study the effect of sequence changes in these structures, since changes would also affect transcription termination. Here, we obtained evidence that even weaker structures, such as eSL1 and eSL2, are capable of affecting RNase $\mathrm{J}^{1} 5^{\prime}$-to- $3^{\prime}$ processing. The fact that the pattern of $3^{\prime}$-end-containing intermediates was the same, whether or not $\Delta e r m C$ mRNA was translated (Fig. 3A), was somewhat surprising, since translation made a significant difference in the effect of SSL $>11$ (Fig. 1E). Perhaps the process of tracking in the $5^{\prime}$-to- $3^{\prime}$ direction and the process of degradation in the $5^{\prime}$-to- $3^{\prime}$ direction relate differently to the flow of ribosomes. More experiments are needed to address this issue.

The $3^{\prime}$-end-containing fragments were visible in the mutant strain with reduced RNase J1 levels, but not in the wild-type strain (Fig. 2A). We hypothesize that the wild-type level of RNase J1 is enough to cause rapid degradation of such fragments. When the level of RNase $\mathrm{J} 1$ is reduced, the endonucleolytic cleavages, which are required only once per molecule to initiate decay, still occur at a wild-type or near wild-type rate, but the impeded processivity of RNase $\mathrm{J} 1$ in the $5^{\prime}$-to- $3^{\prime}$ direction by secondary structure may cause RNase $\mathrm{J} 1$ to release the substrate, and only when high levels of RNase J1 are present does the continual binding and $5^{\prime}$-to- $3^{\prime}$ degradation ensure rapid removal of the $3^{\prime}$-end-containing fragments.

The "bands" detected on Northern blots from lowresolution gels were shown to be a large group of bands on Northern blotting from a high-resolution gel (Fig. 2B). Since the intensity of these bands was much reduced for $\Delta e r m C$ mutants with sequence changes that disrupted eSL1 or eSL2, it is likely that these structures are somewhat resistant to RNase J1 5' exonuclease processivity. However, our mapping of these bands is not entirely consistent with a fall-off of RNase J1 upstream of the structure. Rather, the bands map part way up the $5^{\prime}$ side of the structure itself. Perhaps the slowing of RNase J1 processivity caused by these structures increases the likelihood of substrate release, and such release can occur several nucleotides downstream from where processivity begins to be affected. Future studies will look at the effects of a number of secondary structures predicted to form in vivo, as well as RNase J1 activity on eSL1 and eSL2 in vitro.

The increased intensity of bands 60-73 nt long in the NotI mutant construct, representing 3 ' decay intermediates with $5^{\prime}$ ends between 187 and $200 \mathrm{nt}$, was of interest. The inserted GC-rich sequence, which constitutes the NotI site in the DNA, starts at nucleotide 189. We have found in studies on $\operatorname{trp}$ leader RNA that the GC-rich sequence of a NotI site is inhibitory to PNPase processivity in the $3^{\prime}$-to- $5^{\prime}$ direction, both in vivo and in vitro (Deikus and Bechhofer 2009). We speculate that this particular sequence is also inhibitory to RNase $\mathrm{J} 1$ processivity in the $5^{\prime}$-to- $3^{\prime}$ di- rection. Therefore, disrupting the eSL2 structure eliminates decay intermediates with $5^{\prime}$ ends between 165 and 180 , which we hypothesize are due to partial blockage to RNase J1 processivity, but results in intensification of decay intermediates with $5^{\prime}$ ends between 187 and 200 (Fig. 2B). Again, further in vitro studies with purified RNase J1 are required to test this.

The deletion construct analysis (Fig. 4) suggested that there are at least three preferred RNase J1 target sites located in the downstream half of $\Delta e r m C$ mRNA. These are located upstream of eSL1, between eSL1 and eSL2, and between eSL2 and the $3^{\prime}$ transcription terminator. Deletion of all these three target sites resulted in increased mRNA half-life ( $\sim 15 \mathrm{~min}$ ) (Fig. $4 \mathrm{G})$. RNase J1 cleavage sites that have been mapped in other RNAs occur between or near regions of structured RNA, so the presence of eSL1 and eSL2 may render the AU-rich sequences near these structures preferred target sites. Nevertheless, the stability of the RNA encoded by this deletion construct was further enhanced in an RNase J1 conditional mutant, where the half-life was $>40$ min (Fig. 4G). It is possible that increased stability conferred by the 129-nt deletion is due to elimination of only one element in the RNase $\mathrm{J} 1$ decay pathway. Other elements could include secondary RNase J1 targets in the upstream half of the mRNA, as well as exonucleolytic decay from the $5^{\prime}$ end. It will be interesting to determine half-lives of $\Delta \mathrm{ermC}$ mRNA constructs that combine $5^{\prime}$ stabilizer sequences at the $5^{\prime}$ end (Sharp and Bechhofer 2005) and the 129-nt deletion, to see whether even such constructs are further stabilized in an RNase J1 conditional mutant.

\section{MATERIALS AND METHODS}

\section{Bacterial strains}

The B. subtilis host was BG1, which is trpC2 thr-5. Chromosomal DNA from the RNase J1 conditional mutant strain (Britton et al. 2007) was used to transform BG1 to Em resistance. The RNase J1 conditional strain also contained plasmid pMAP65, which carries extra copies of the lacI gene (Petit et al. 1998). The preparation and transformation of B. subtilis competent cell cultures were as described previously (Dubnau and Davidoff-Abelson 1971). E. coli strain DH5 $\alpha$ (Grant et al. 1990) was the host for plasmid constructions.

\section{Plasmids}

The wild-type $\Delta$ erm $C$ gene was carried on plasmid pYH250, an E. coli/B. subtilis shuttle plasmid that contains a chloramphenicol resistance marker (Sharp and Bechhofer 2005). In pYH250, a HindIII site is present at the beginning of the $\Delta e r m C$ transcriptional unit and an EcoRI site is located downstream from the $\Delta e r m C$ transcription terminator. The HindIII site was incorporated into mutagenic PCR primers that included nucleotide sequence changes in the $\Delta e r m C$ CDS. These $5^{\prime}$ primers were used in conjunction with a $3^{\prime}$ primer containing the downstream EcoRI 
site to amplify $\Delta$ erm $C$, giving an amplicon that was cloned as a HindIII-EcoRI fragment into the pYH250 vector. For some constructs, a mutagenic oligonucleotide that contained the HpaI site consisting of codons 17 and 18 was used in conjunction with a primer that contained the $\mathrm{XbaI}$ site located about 600 base pairs upstream of $\Delta e r m C$. For construction of the NotI and ClaI mutants (Fig. 2C), complementary mutagenic oligonucleotides containing the mutated sequence were used in separate PCR reactions with an oligonucleotide containing either the upstream $X b a I$ sequence or the downstream EcoRI sequence. The two PCR amplicons were annealed to each other and were amplified in a second round with the upstream and downstream primers. The mutated fragment was digested with $\mathrm{Xba \textrm {I }}$ and EcoRI and used to replace the small $X b a \mathrm{I}-E c o$ RI fragment of pYH250.

\section{RNA analysis}

Supplemented minimal medium for RNA isolation, Northern blot analysis, and 5S rRNA probing as a quantitation control were as described (Oussenko et al. 2005). For expression of RNase J1 in the conditional mutant strain, IPTG was added to $1 \mathrm{mM}$. For the analysis of steady-state RNA shown in Figure 2A,B, strains were grown in $2 \times \mathrm{YT}$ medium ( $1 \%$ yeast extract, $2 \%$ tryptone, $1 \% \mathrm{NaCl}$ ), in the absence of IPTG. For induction of ribosome stalling (Fig. 3B), Em was added to a concentration of $5.0 \mu \mathrm{g} / \mathrm{mL}$. Quantitation of fulllength $\Delta e r m C$ mRNA and RNA processing products was done with a Storm 860 PhosphorImager (Molecular Dynamics) or a Typhoon TRIO variable mode imager (GE Healthcare).

\section{ACKNOWLEDGMENTS}

This work was supported by Public Health Service grant GM48804 from the National Institutes of Health.

Received May 22, 2009; accepted August 24, 2009.

\section{REFERENCES}

Bechhofer DH. 2009. Chapter 6 messenger RNA decay and maturation in Bacillus subtilis. Prog Nucleic Acid Res Mol Biol 85: 231-273.

Bechhofer DH, Dubnau D. 1987. Induced mRNA stability in Bacillus subtilis. Proc Natl Acad Sci 84: 498-502.

Bechhofer DH, Zen KH. 1989. Mechanism of erythromycin-induced ermC mRNA stability in Bacillus subtilis. J Bacteriol 171: 5803-5811.

Britton RA, Wen T, Schaefer L, Pellegrini O, Uicker WC, Mathy N, Tobin C, Daou R, Szyk J, Condon C. 2007. Maturation of the $5^{\prime}$ end of Bacillus subtilis 16S rRNA by the essential ribonuclease YkqC/RNase J1. Mol Microbiol 63: 127-138.

Brosius J. 1992. Compilation of superlinker vectors. Methods Enzymol 216: 469-483.

Celesnik H, Deana A, Belasco JG. 2007. Initiation of RNA decay in Escherichia coli by $5^{\prime}$ pyrophosphate removal. Mol Cell 27: 79-90.

Condon C. 2003. RNA processing and degradation in Bacillus subtilis. Microbiol Mol Biol Rev 67: 157-174.

Condon C. 2007. Maturation and degradation of RNA in bacteria. Curr Opin Microbiol 10: 271-278.

Daou-Chabo R, Mathy N, Benard L, Condon C. 2009. Ribosomes initiating translation of the hbs mRNA protect it from $5^{\prime}$-to- $3^{\prime}$ exoribonucleolytic degradation by RNase J1. Mol Microbiol 71: $1538-1550$.

Deana A, Celesnik H, Belasco JG. 2008. The bacterial enzyme RppH triggers messenger RNA degradation by $5^{\prime}$ pyrophosphate removal. Nature 451: 355-358.
Deikus G, Bechhofer DH. 2007. Initiation of decay of Bacillus subtilis trp leader RNA. J Biol Chem 282: 20238-20244.

Deikus G, Bechhofer DH. 2009. Bacillus subtilis trp leader RNA: RNase $\mathrm{J} 1$ endonuclease cleavage specificity and PNPase processing. J Biol Chem 284: 26394-26401.

Deikus G, Condon C, Bechhofer DH. 2008. Role of Bacillus subtilis RNase J1 endonuclease and $5^{\prime}$-exonuclease activities in trp leader RNA turnover. J Biol Chem 283: 17158-17167.

DiMari JF, Bechhofer DH. 1993. Initiation of mRNA decay in Bacillus subtilis. Mol Microbiol 7: 705-717.

Drider D, DiChiara JM, Wei J, Sharp JS, Bechhofer DH. 2002. Endonuclease cleavage of messenger RNA in Bacillus subtilis. Mol Microbiol 43: 1319-1329.

Dubnau D. 1984. Translational attenuation: The regulation of bacterial resistance to the macrolide-lincosamide-streptogramin B antibiotics. CRC Crit Rev Biochem 16: 103-132.

Dubnau D, Davidoff-Abelson R. 1971. Fate of transforming DNA following uptake by competent Bacillus subtilis. I. Formation and properties of the donor-recipient complex. J Mol Biol 56: 209-221.

Even S, Pellegrini O, Zig L, Labas V, Vinh J, Brechemmier-Baey D, Putzer H. 2005. Ribonucleases J1 and J2: Two novel endoribonucleases in B. subtilis with functional homology to E. coli RNase E. Nucleic Acids Res 33: 2141-2152.

Grant SG, Jessee J, Bloom FR, Hanahan D. 1990. Differential plasmid rescue from transgenic mouse DNAs into Escherichia coli methylationrestriction mutants. Proc Natl Acad Sci 87: 4645-4649.

Hunt A, Rawlins JP, Thomaides HB, Errington J. 2006. Functional analysis of 11 putative essential genes in Bacillus subtilis. Microbiology 152: 2895-2907.

Li de la Sierra-Gallay I, Zig L, Jamalli A, Putzer H. 2008. Structural insights into the dual activity of RNase J. Nat Struct Mol Biol 15: 206-212.

Mader U, Zig L, Kretschmer J, Homuth G, Putzer H. 2008. mRNA processing by RNases $\mathrm{J} 1$ and $\mathrm{J} 2$ affects Bacillus subtilis gene expression on a global scale. Mol Microbiol 70: 183-196.

Mathy N, Benard L, Pellegrini O, Daou R, Wen T, Condon C. 2007. 5'-To-3' exoribonuclease activity in bacteria: Role of RNase J1 in rRNA maturation and 5' stability of mRNA. Cell 129: 681-692.

Ohki R, Tateno K. 2004. Increased stability of bmr3 mRNA results in a multidrug-resistant phenotype in Bacillus subtilis. J Bacteriol 186: 7450-7455.

Oussenko IA, Abe T, Ujiie H, Muto A, Bechhofer DH. 2005. Participation of $3^{\prime}$-to-5' exoribonucleases in the turnover of Bacillus subtilis mRNA. J Bacteriol 187: 2758-2767.

Petit MA, Dervyn E, Rose M, Entian KD, McGovern S, Ehrlich SD, Bruand C. 1998. PcrA is an essential DNA helicase of Bacillus subtilis fulfilling functions both in repair and rolling-circle replication. Mol Microbiol 29: 261-273.

Sharp JS, Bechhofer DH. 2003. Effect of translational signals on mRNA decay in Bacillus subtilis. J Bacteriol 185: 5372-5379.

Sharp JS, Bechhofer DH. 2005. Effect of 5'-proximal elements on decay of a model mRNA in Bacillus subtilis. Mol Microbiol 57: 484495.

Vazquez-Laslop N, Thum C, Mankin AS. 2008. Molecular mechanism of drug-dependent ribosome stalling. Mol Cell 30: 190-202.

Weisblum B. 1985. Inducible resistance to macrolides, lincosamides, and streptogramin type B antibiotics: The resistance phenotype, its biological diversity, and structural elements that regulate expression-a review. J Antimicrob Chemother 16 Suppl A: 63-90.

Yao S, Bechhofer DH. 2009. Processing and stability of inducibly expressed $r p s O$ mRNA derivatives in Bacillus subtilis. J Bacteriol 191: 5680-5689.

Yao S, Blaustein JB, Bechhofer DH. 2007. Processing of Bacillus subtilis small cytoplasmic RNA: Evidence for an additional endonuclease cleavage site. Nucleic Acids Res 35: 4464-4473.

Yao S, Blaustein JB, Bechhofer DH. 2008. Erythromycin-induced ribosome stalling and RNase J1-mediated mRNA processing in Bacillus subtilis. Mol Microbiol 69: 1439-1449. 

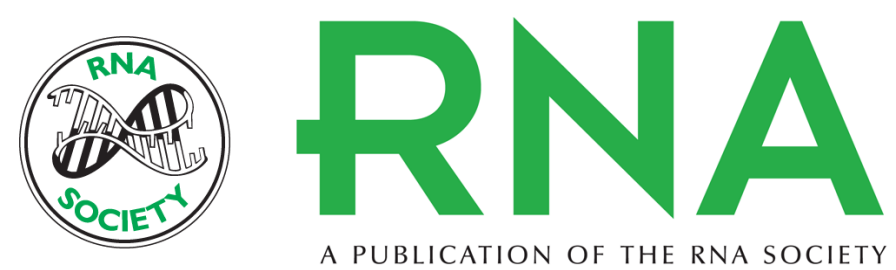

\section{Bacillus subtilis RNase $\mathrm{J} 1$ endonuclease and $5^{\prime}$ exonuclease activities in the turnover of $\triangle e r m C$ mRNA}

Shiyi Yao, Josh S. Sharp and David H. Bechhofer

RNA 2009 15: 2331-2339 originally published online October 22, 2009

Access the most recent version at doi:10.1261/rna.1749109

References This article cites 32 articles, 11 of which can be accessed free at: http://rnajournal.cshlp.org/content/15/12/2331.full.html\#ref-list-1

License

Email Alerting Receive free email alerts when new articles cite this article - sign up in the box at the Service top right corner of the article or click here. 